\title{
Comparing the efficacy of four different protocols for eradicating of Helicobacter pylori infection in Ahvaz, southwest Iran
}

\author{
Abdolrahim Masjedizadeh ${ }^{1}$, Narjes Zaeemzadeh², Seyyed Ali Mard ${ }^{1}$, Ghazal Salehi Vanani $^{1}$ \\ ${ }^{1}$ Research Center for Infectious Diseases of Digestive System, Ahvaz Jundishapur University of Medical Sciences, Ahvaz, Iran \\ ${ }^{2}$ Research Center for Infectious Diseases of Digestive System, Departement of Pharmacology, Ahvaz Jundishapur University \\ of Medical Sciences, Ahvaz, Iran
}

Prz Gastroenterol 2015; 10 (2): 94-99

DOI: $10.5114 / p g .2015 .49001$

Key words: Helicobacter pylori, Ahvaz, clarithromycin, bismuth subcitrate, metronidazol, ciprofloxacin.

Address for correspondence: Prof. Abdolrahim Masjedizadeh, Research Center for Infectious Diseases of Digestive System, Ahvaz Jundishapour University of Medical Sciences, Golestan, 6155837539 Ahvaz, Iran, phone: +989161114970, fax: +986113910642, e-mail: masjedi-r@ajums.ac.ir

\begin{abstract}
Introduction: Helicobacter pylori (H. pylori) is the common cause of many gastrointestinal diseases, especially peptic ulcer. Therefore, a successful treatment of this infection decreases the financial burden on health systems.

Aim: Different combinations of antibiotics are used for the eradication of this bacterium worldwide. The goal of this study is to compare the efficacy of four different protocols used for this purpose in Ahvaz.

Material and methods: A total number of 400 patients with $\mathrm{H}$. pylori infection were randomly divided into four groups (100 in each): (1) OAC: omeprazole (20 mg/b.i.d.), amoxicillin (1000 mg/b.i.d.), clarithromycin (500 mg/b.i.d.) for 10 days. (2) OCF: omeprazole (20 mg/b.i.d.), ciprofloxacin (500 mg/b.i.d.), furazolidone (100 mg/b.i.d.) for 10 days. (3) OBAM: omeprazole (20 mg/b.i.d.), bismuth subcitrate (240 mg/b.i.d.), amoxicillin (1000 mg/b.i.d.), metronidazol (500 mg/b.i.d.) for 14 days. (4) OBTM: omeprazole (20 mg/b.i.d.), bismuth subcitrate ( $240 \mathrm{mg} /$ b.i.d.), tetracycline (500 mg/b.i.d.), metronidazol (500 mg/b.i.d.) for 14 days. At the end the viability of the bacterium was assessed by $\mathrm{C}^{14}$ urea breath test.

Results: The rate of $\mathrm{H}$. pylori eradication was $92 \%, 59 \%, 73 \%$, and $76 \%$ in OAC, OCF, OBAM, and OBTM groups, respectively (based on intention to treat analysis). The eradication rate was $93.9 \%, 62.1 \%, 77.7 \%$, and $84.4 \%$ in OAC, OCF, OBAM, and OBTM groups, respectively (based on per protocol analysis). There was a statistically significant increase in eradication rate in the OAC group in comparison with the others $(p<0.001)$.

Conclusions: Standard triple therapy (omeprazole, amoxicillin, clarithromycin) remains the most effective regimen for H. pylori eradication in Ahvaz.
\end{abstract}

\section{Introduction}

Helicobacter pylori (H. pylori) was introduced to scientific society in the 1980 s by Marshal and Warren, when they announced that the bacterium can be a cause for active chronic gastritis and peptic ulcer disease [1]. Since that time research was started on the subject and to date it has shown that the microorganism can cause several diseases involving the gastrointestinal tract, including acute and chronic gastritis, atrophic gastritis, peptic ulcer disease, gastric mucosa-associated lymphoid tissue (MALT) lymphoma, and gastric adenocarcinoma [2]. Among extraintesti- nal diseases, the bacillus has been correlated to Henoch-Schonlein purpura [3], unexplained iron-deficiency anaemia [4, 5], and idiopathic thrombocytopenic purpura (ITP) [6], and its eradication has been recommended in these cases. The eradication of $\mathrm{H}$. pylori is also associated with decreasing the rate of gastrointestinal bleeding in users of nonsteroidal anti-inflammatory drugs (NSAIDs) [7]. Therefore, eradication of the infection can result in different levels of decreasing morbidity and mortality generally. It is documented that successful eradication of the bacterium can make the patient medication free, even in complicated peptic ulcer cases [8]. 
Various guidelines for the management of H. pylori infection are available worldwide. Among these, the latest one is the "Maastricht IV/ Florence Consensus Report" (2012), which was written by the European Helicobacter Study Group and suggests various treatment regimens, including: standard triple therapy (STT) with proton pump inhibitor (PPI)-clarithromycin and amoxicillin or metronidazole, bismuth-containing quadruple therapy, levofloxacin-containing triple therapy, and non-bismuth quadruple treatment (sequential (SQT) or concomitant). Sequential quadruple treatment includes a 5-day period with PPI-amoxicillin, followed by a 5-day period with PPI-clarithromycin-metronidazole (or tinidazole), and finally, treatments based on antimicrobial susceptibility testing [9].

Because no new drug has been developed for this infection, a number of research projects have been carried out in recent years using different combinations of known antibiotics.

\section{Aim}

In this study we aimed to compare the efficacy of four protocols for $H$. pylori infection eradication: (1) omeprazole, amoxicillin, clarithromycin (OAC), (2) omeprazole, ciprofloxacin, furazolidone (OCF), (3) omeprazole, bismuth subcitrate, amoxicillin, metronidazol (OBAM), (4) omeprazole, bismuth subcitrate, tetracycline, and metronidazol (OBTM) in Ahvaz, southwest Iran.

\section{Material and methods}

\section{Subjects}

This randomised clinical trial was carried out on 400 patients with peptic complications, who referred to governmental gastroenterology clinics of Ahvaz (southwest of Iran) between January and September 2010. Simple random selection of patients was carried out on group stratification based on age and sex. All patients were candidates for upper gastrointestinal (GI) endoscopy. The $H$. pylori infection was proven by a positive rapid urease test (RUT, $72.1 \%$ of patients) or a positive histopathological examination (27.9\% of patients) of the biopsy specimens obtained from stomach antrum and corpus.

The exclusion criteria included: a history of G6PD deficiency, previous gastric surgery, drug allergies, chronic hepatic, renal and pulmonary disease, gastrointestinal malignancies, taking drugs such as antibiotics, gastric acid inhibitors, NSAIDs during the last month, pregnancy, and breast feeding.

The study was approved by the ethical committee of the Ahvaz Jundishapour University of Medical Sciences. All volunteers signed an informed written consent form before entering the study.

\section{Study design}

Each 100 consecutive patients were enrolled to receive one of the four different protocols described below: (1) OAC group: omeprazole (20 mg/b.i.d.), amoxicillin (1000 mg/b.i.d.), clarithromycin (500 mg/b.i.d.) for 10 days. (2) OCF group: omeprazole (20 mg/b.i.d.), ciprofloxacin (500 mg/b.i.d.), furazolidone (100 mg/ b.i.d.) for 10 days. (3) OBAM group: omeprazole $(20 \mathrm{mg} /$ b.i.d.), bismuth subcitrate (240 mg/b.i.d.), amoxicillin (1000 mg/b.i.d.), metronidazol (500 mg/b.i.d.) for 14 days. (4) OBTM group: omeprazole (20 mg/b.i.d.), bismuth subcitrate $(240 \mathrm{mg} /$ b.i.d.), tetracycline (500 mg/b.i.d.), metronidazol (500 mg/b.i.d.) for 14 days.

After accomplishing the intervention period, the patients' compliance and appearance of side effects were assessed via an interview.

The patient's compliance was categorized as follows: good (taking $80 \%$ of the drugs in the regimen), fair (taking $60-80 \%$ of the drugs), and poor (taking less than $60 \%$ of the drugs). Patients with poor compliance were omitted from the study.

In terms of presenting side effects, the patients were categorised as follows: mild (no interference with daily activities), moderate (mild interference with daily activities), and severe (completely stopped daily activities).

All patients made an appointment for $\mathrm{C}^{14}$ urea breath test ( $\left.C^{14} \cup B T\right) 8$ weeks after the end of study in order to avoid false negative findings [10]. Patients were advised to stop using any antibiotics, gastric acid inhibitors, or bismuth subcitrate 2 weeks prior to the test.

\section{Assessment of Helicobacter pylori status}

Biopsy specimens were taken during upper gastrointestinal endoscopy either for rapid urease test (CLO test, Campylobacter-like organism test) (Ballard Medical Products, Draper, UT, USA) or for histological assessment. In the latter case, the specimens were fixed in 10\% formalin and stained with haematoxylin-eosin and Giemsa stain.

$C^{14} U B T$ was used to evaluate the $H$. pylori viability at the end of study. Patients who did not take the test were excluded from the study. The test was based upon the ability of $H$. pylori to convert urea to ammonia and carbon dioxide [11].

The test was done 30 min after oral administration of the capsules containing $\mathrm{C}^{14}$ urea $(37 \mathrm{kBq})$. Patients exhaled in $\mathrm{KOH}$ solution until it became colourless, which was the sign that $1 \mathrm{mmol}$ of $\mathrm{CO}_{2}$ had been trapped. After adding $15 \mathrm{ml}$ of scintillation fluid, the radioactivity in the collected samples was measured by liquid scintillation counter (LKB Wallac - Rack Beta Spectral) [12].

The test was considered as negative if the amount of $\mathrm{CO}_{2}$ was less than 50 , and a negative test was considered a successful treatment. 


\section{Statistical analysis}

Data were analysed using $\chi^{2}$ statistical test. Value of $p<0.05$ was considered as significant.

\section{Results}

\section{Demographic data}

A total of 400 patients (211 males, 189 females) were recruited (Table I). Each consecutive 100 patients were randomly entered to one of the groups: OAC, OCF, OBAM, and OBTM. Twenty-three patients $(2,5,6$, and 10 patients from the OAC, OCF, OBAM, and OBTM groups, respectively) were totally excluded from the per protocol analysis. The reasons for exclusion were side effects occurrence $(1,3,2$, and 5 cases from the OAC, OCF, OBAM, and OBTM groups, respectively) and missing the follow-up program $(1,2,4$, and 5 cases from the OAC, OCF, OBAM, and OBTM groups, respectively). None of the patients had poor compliance. At the end of the study, a total of 377 patients were analysed in per protocol analysis (Figure 1). The most common chief complaints for being referred to the gastrointestinal clinic and undergoing an upper GI endoscopy were epigastric pain and dyspepsia/heartburn in decreasing order of frequency in all treatment groups. Flatulence and strong family history of gastric cancer were the other common symptoms. The most common endoscopic findings were gastritis, and normal and duodenal ulcer, in all treatment groups, but the OBTM group, which showed gastritis, erosive gastritis, and duodenal ulcer as common endoscopic features. The side-effect profile in the different groups is shown in Table II. The most common side effects in the OAC, OCF, OBAM, and OBTM groups were dizziness/taste perversion, nausea/vomiting, dizziness, and taste perversion, respectively. There was no complaint of abdominal pain, constipation, and skin rashes in any of the groups.

\section{$\mathrm{C}^{14}$ urea breath test}

The rate of $H$. pylori eradication (negative result of $\mathrm{C}^{14} \mathrm{UBT}$ ) based on intention to treat analysis (ITT) was $92 \%, 59 \%, 73 \%$, and $76 \%$ in the OAC, OCF, OBAM, and OBTM groups, respectively. Eradication rate based on per protocol (PP) analysis was 93.9\%, 62.1\%, 77.7\%, and $84.4 \%$ in the OAC, OCF, OBAM, and OBTM groups, respectively (Figure 2). There was a statistically significant increase in eradication rate in the OAC group compared to the other ones $(p<0.001)$.

Table I. Demographic characteristics of volunteers

\begin{tabular}{|c|c|c|c|c|c|}
\hline Parameter & OAC & OCF & OBAM & ОВТМ & Total \\
\hline Total number (ITT*) & 100 & 100 & 100 & 100 & 400 \\
\hline Included per-protocol (PP) & 98 & 95 & 94 & 90 & 377 \\
\hline Female, $n(\%)$ & $43(43.9)$ & $50(52.6)$ & $43(45.7)$ & $43(47.8)$ & $179(47.5)$ \\
\hline Male, $n(\%)$ & $55(56.1)$ & $45(47.4)$ & $51(54.3)$ & $47(52.2)$ & $198(52.5)$ \\
\hline $\begin{array}{l}\text { Age range (mean } \pm \text { SD) } \\
\text { [years] }\end{array}$ & $10-70(40 \pm 13.1)$ & $14-80(41 \pm 13.18)$ & $16-73(38 \pm 11.79)$ & $15-79(39 \pm 12.53)$ & \\
\hline
\end{tabular}

"Intention to treat. There was no significant difference between the groups regarding sex and age distribution $(p=0.65 \%)$.

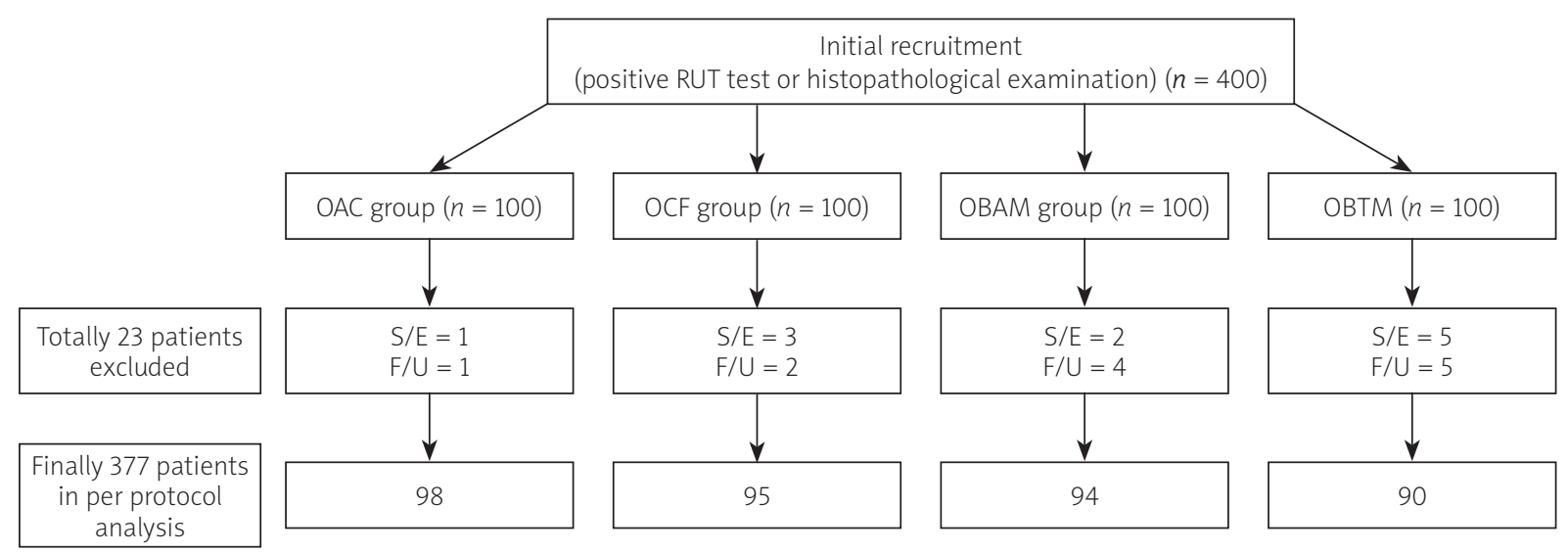

Figure 1. Study design 
Table II. Adverse effects

\begin{tabular}{|c|c|c|c|c|}
\hline Side effect & OAC, $n(\%)$ & OCF, $n(\%)$ & OBAM, $n(\%)$ & OBTM, $n(\%)$ \\
\hline Abdominal pain & - & - & - & - \\
\hline Constipation & - & - & - & - \\
\hline Diarrhoea & $5(5.1)$ & $1(1.05)$ & $2(2.12)$ & - \\
\hline Dizziness & $10(10.2)$ & $1(1.05)$ & $7(7.44)$ & $2(2.22)$ \\
\hline Taste perversion & $10(10.2)$ & - & $5(5.31)$ & $6(6.66)$ \\
\hline Headache & $3(3.06)$ & $2(2.1)$ & $4(4.25)$ & - \\
\hline Anorexia & $3(3.06)$ & $5(5.26)$ & $4(4.255)$ & - \\
\hline Nausea/vomiting & $4(4.08)$ & $12(12.63)$ & $2(2.12)$ & $2(2.12)$ \\
\hline Skin rash & - & - & - & - \\
\hline Others & $1(1.02)$ & - & - & 1 (1.11) \\
\hline Total & $36(36.73)$ & $21(22.1)$ & $22(23.4)$ & $9(10)$ \\
\hline
\end{tabular}

\section{Discussion}

In this study the eradication rates of $H$. pylori in patients with a documented infection were compared using four different regimens: OAC: omeprazole, amoxicillin, clarithromycin; OCF: omeprazole, ciprofloxacin, furazolidone; OBAM: omeprazole, bismuth subcitrate, amoxicillin, metronidazol; and OBTM: omeprazole, bismuth subcitrate, tetracycline, metronidazol. The rates of eradication achieved in this study were acceptable apart from the OCF regimen, according to a pooled-data analysis on this issue, which showed that a rate of $>75 \%$ eradication is an acceptable rate [13].

In the current study we showed that the eradication was more effective with standard triple therapy (STT, a PPI-clarithromycin and amoxicillin) regimen in comparison to the other three regimens. Standard triple therapy remains the first line treatment for eradication of $H$. pylori around the world, unless the area in question has a resistance rate above 15-20\% [9]. However, Shiota and Yamaoka recently reported that quadruple therapy should be replaced by clarithromycin-based triple therapy as initial therapy for eradication of this bacterium [14].

The sole study on $\mathrm{H}$. pylori resistance rate in Ahwaz showed that the rate of clarithromycin resistance in this city is $24 \%$, which places the city in the category of regions with high resistance to clarithromycin [15]. Also Mohammadi et al. in 2003 already warned that a near $20 \%$ rate of clarithromycin resistance should alert to the frequency of macrolide resistant strains in Iran [16]. According to the evidence mentioned above, the STT should not be used as a treatment regimen for $H$. pylori eradication in Ahvaz. But this regimen is still used, may-

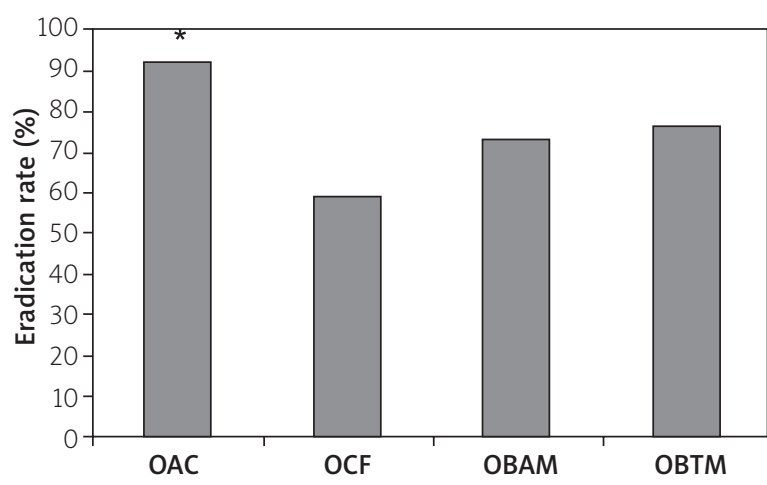

Figure 2. Eradication rate of $H$. pylori in four different groups based on per protocol analysis (\%)

*Value of $p<0.05$ indicates significant difference versus OCF, OBAM and OBTM. OAC - omeprazole, amoxicillin, clarithromycin; OCF - omeprazole, ciprofloxacin, furazolidone; OBAM omeprazole, bismuth subcitrate, amoxicillin, metronidazole; OBTM - omeprazole, bismuth subcitrate, tetracycline, and metronidazole.

be due to the lack of any other report on this issue. Albeit, researche on the entity of clarithromycin resistance rate in areas of Iran do not show parallel results; some report that the rate of clarithromycin resistance is under the threshold mentioned earlier [17, 18], and some report a rate of resistance exactly within the threshold range of $15-20 \%$ or very close to it [19-21]. The results of another study performed in Ahvaz in 2007 by Hajiani et al. also confirm our results in this respect, showing that the STT regimen is more powerful than a quadruple therapy in the eradication of this infection; this shows that the high rate of clarithromycin resistance in this region can be overcome by combination STT [22]. The 
rate of eradication was $92 \%$ with the OAC regimen in our study, which is confirmed by at least four studies that reported an eradication rate of $>75 \%$ with this regimen [23-26]. Also, our eradication rate was higher than all these studies, which may refer to the much lower percentage of excluded patients in per protocol analysis in our study (2\%) as compared with $17-23 \%$ in the above-mentioned literature.

In addition, two of these studies used a shorter treatment duration (7 days) than the current study (10 days) $[25,26]$.

There is no report of resistance of $H$. pylori strains to furazolidone and ciprofloxacin in Ahvaz. A study from the north of Iran in 2011 reported the rate of resistance was $34.5 \%$ and $61.4 \%$ for ciprofloxacin and furazolidone, respectively [27]. Another study from a big referral medical centre for paediatrics in Tehran showed a rate of $34.5 \%$ resistance to ciprofloxacin, but it has no data on furazolidone [28]. The results of the latter study can be generalized to Ahvaz, because the place where the study was performed is a big referral centre in Iran, and this may explain why the OCF regimen has a much lower eradication rate compared with the other regimens.

The rate of eradication with the OBTM regimen in our study was $76 \%$, which is higher than the reports by Mantzaris et al. and Gomollon et al. The reason could be due to the shorter treatment period ( 7 and 10 days vs. 14 days in the present study), the smaller sample size (48 and 71 patients vs. 100 patients in the present study), and the higher percentage of excluded patients in per protocol analysis (31.25\% and $35 \%$ vs. $10 \%$ in the current study) $[23,25]$.

However, the eradication rate in our study with the OBTM regimen was lower than that seen in the study by Laine et al. and Malfertheiner et al., which may be due to lower sample size (100 patients in our study vs. 138 and 218 patients, respectively) and lower doses of tetracycline, metronidazole, and bismuth subcitrate [24, 29].

Another point in this study was that the more powerful regimen (OAC) had the higher percentage of total side effect occurrence. The reason can be the fact that the severity of side effects in the OAC group is very low, as only 1 patient in this group (fewer in number in comparison to other groups) left the study because of side effect appearance.

\section{Conclusions}

We have shown that despite heterogeneous reports about the resistance of $\mathrm{H}$. pylori strains to clarithromycin in Iran and the record of a high rate of resistance to clarithromycin in Ahvaz, standard triple therapy (omeprazole, amoxicillin, clarithromycin) remains the most effective regimen for eradicating of $H$. pylori infection in Ahvaz.

\section{Acknowledgments}

The authors would like to thank Ahvaz Jundishpur University of Medical Sciences for providing financial support.

\section{Conflict of interest}

The authors declare no conflict of interest.

\section{References}

1. Marshall B, Warren JR. Unidentified curved bacilli in the stomach of patients with gastritis and peptic ulceration. Lancet 1984; 323: 1311-5.

2. Roesler BM, Costa SC, Zeitune JM. Eradication treatment of Helicobacter pylori infection: its importance and possible relationship in preventing the development of gastric cancer. ISRN Gastroenterology 2012; 2012: 935410.

3. Ulas T, Tursun I, Dal MS, et al. Rapid improvement of Henoch-Schonlein purpura associated with the treatment of Helicobacter pylori infection. J Res Med Sci 2012; 17: 1086-8.

4. Qu XH, Huang XL, Xiong P, et al. Does Helicobacter pylori infection play a role in iron deficiency anemia? A meta-analysis. World J Gastroenterol 2010; 16: 886-96.

5. Muhsen K, Cohen D. Helicobacter pylori infection and iron stores: a systematic review and meta-analysis. Helicobacter 2008; 13: 323-40.

6. Arnold DM, Bernotas A, Nazi I, et al. Platelet count response to H. pylori treatment in patients with immune thrombocytopenic purpura with and without $\mathrm{H}$. pylori infection: a systematic review. Haematologica 2009; 94: 850-6.

7. Roug S, Madsen LG. Importance of post-treatment follow-up to secure sufficient eradication therapy for Helicobacter pylori. Dan Med J 2012; 59: A4553.

8. Gisbert JP, Calvet X, Cosme A, et al. Long-term follow-up of 1,000 patients cured of Helicobacter pylori infection following an episode of peptic ulcer bleeding. Am J Gastroenterol 2012; 107: 1197-204.

9. Malfertheiner P, Megraud F, O'Morain CA, et al. Management of Helicobacter pylori infection: the Maastricht IV/Florence Consensus Report. Gut 2012; 61: 646-64.

10. Bravo LE, Realpe JL, Campo C, et al. Effects of acid suppression and bismuth medications on the performance of diagnostic tests for Helicobacter pylori infection. Am J Gastroenterol 1999; 94: 2380-3.

11. Chey WD, Wong BC. American College of Gastroenterology guideline on the management of Helicobacter pylori infection. Am J Gastroenterol 2007; 102: 1808-25.

12. Artiko VM, Obradovic V, Petrovic N, et al. 14C-urea breath test in the detection of Helicobacter pylori infection. Nucl Med Rev Cent East Eur 2001; 4: 101-3.

13. Zullo A, De Francesco V, Hassan C, et al. The sequential therapy regimen for Helicobacter pylori eradication: a pooled-data analysis. Gut 2007; 56: 1353-7.

14. Shiota S, Yamaoka Y. Strategy for the treatment of Helicobacter pylori infection. Curr Pharm Des 2014; 20: 4489-500.

15. Moosavian M, Tajbakhsh S, Samarbaf-Zadeh AR. Rapid detection of clarithromycin-resistant Helicobacter pylori in patients with dyspepsia by fluorescent in situ hybridization (FISH) compared with the E-test. Ann Saudi Med 2006; 27: 84-8. 
16. Mohammadi M, Doroud D, Massarrat S, et al. Clarithromycin resistance in Iranian $\mathrm{H}$. pylori strains before introduction of clarithromycin. Helicobacter 2003; 8: 80.

17. Kohanteb J, Bazargani A, Saberi-Firoozi M, et al. Antimicrobial susceptibility testing of Helicobacter pylori to selected agents by agar dilution method in Shiraz-Iran. Indian J Med Microbiol 2007; 25: 374-7.

18. Siavashi F, Safari F, Doratotaj D, et al. Antimicrobial resistance of Helicobacter pylori isolates from Iranian adults and children. Arch Iran Med 2006; 9: 308-14.

19. Mirzaei N, Poursina F, Faghri J, et al. Prevalence of resistance to Helicobacter pylori strains to selected antibiotics in Isfahan, Iran. Jundishapur J Microbiol 2013; 6: e6342.

20. Khademi F, Faghri J, Poursina F, et al. Resistance pattern of Helicobacter pylori strains to clarithromycin, metronidazole, and amoxicillin in Isfahan, Iran. J Res Med Sci 2013; 18: 1056 60.

21. Mohammadi M, Doroud D, Mohajerani N, et al. Helicobacter pylori antibiotic resistance in Iran. World J Gastroenterol 2005; 11: 6009-13.

22. Hajiani E, Hashemi J, Vosoghi T. Comparison of a 10 day triple and a two-week quadruple therapy in eradicating Helicobacter pylori infection in patients referred to Imam Khomeini Hospital Clinics Ahwaz, Iran. Jundishapur Journal of Natural Pharmaceutical Products 2007; 3: 45-52.

23. Mantzaris GJ, Petraki K, Archavlis E, et al. Omeprazole triple therapy versus omeprazole quadruple therapy for healing duodenal ulcer and eradication of Helicobacter pylori infection: a 24-month follow-up study. Eur J Gastroenterol Hepatol 2002; 14: 1237-43.

24. Laine L, Hunt R, El-Zimaity $\mathrm{H}$, et al. Bismuth-based quadruple therapy using a single capsule of bismuth biskalcitrate, metronidazole, and tetracycline given with omeprazole versus omeprazole, amoxicillin, and clarithromycin for eradication of Helicobacter pylori in duodenal ulcer patients: a prospective, randomized, multicenter, North American trial. Am J Gastroenterol 2003; 98: 562-7.

25. Gomollón F, Valdepérez J, Garuz R, et al. Cost-effectiveness analysis of 2 strategies of Helicobacter pylori eradication: results of a prospective and randomized study in primary care [Spain]. Med Clin 2000; 115: 1-6.

26. Calvet X, Ducons J, Guardiola J, et al. One-week triple vs. quadruple therapy for Helicobacter pylori infection - a randomized trial. Aliment Pharmacol Ther 2002; 16: 1261-7.

27. Abadi ATB, Taghvaei T, Mobarez AM, et al. Frequency of antibiotic resistance in Helicobacter pylori strains isolated from the northern population of Iran. J Microbiol 2011; 49: 987-93.

28. Falsafi T, Mobasheri F, Nariman F, et al. Susceptibilities to different antibiotics of Helicobacter pylori strains isolated from patients at the pediatric medical center of Tehran, Iran. J Clin Microbiol 2004; 42: 387-9.

29. Malfertheiner P, Bazzoli F, Delchier JC, et al. Helicobacter pylori eradication with a capsule containing bismuth subcitrate potassium, metronidazole, and tetracycline given with omeprazole versus clarithromycin-based triple therapy: a randomised, open-label, non-inferiority, phase 3 trial. Lancet 2011; 377: 905-13.

Received: 6.07.2014

Accepted: 11.10 .2014 